University of Wollongong

Research Online

Faculty of Engineering and Information

Faculty of Engineering and Information

Sciences - Papers: Part B

Sciences

2019

A survey on what Australians with upper limb difference want in a prosthesis: justification for using soft robotics and additive manufacturing for customized prosthetic hands

\author{
Benjamin Stephens-Fripp \\ University of Wollongong, bsf147@uowmail.edu.au \\ Mary Jean Walker \\ Monash University \\ Eliza Goddard \\ University of Tasmania, University of New South Wales \\ Gursel Alici \\ University of Wollongong, gursel@uow.edu.au
}

Follow this and additional works at: https://ro.uow.edu.au/eispapers1

Part of the Engineering Commons, and the Science and Technology Studies Commons

Research Online is the open access institutional repository for the University of Wollongong. For further information contact the UOW Library: research-pubs@uow.edu.au 


\title{
A survey on what Australians with upper limb difference want in a prosthesis: justification for using soft robotics and additive manufacturing for customized prosthetic hands
}

\author{
Abstract \\ Purpose: Upper limb prostheses are part of a rapidly changing market place. Despite development in \\ device design, surveys report low levels of uptake and dissatisfaction with current prosthetic design. In \\ this study, we present the results of a survey conducted with people with upper limb difference in \\ Australia on their use of current prostheses and preferences in a prosthetic in order to inform future \\ prosthetic hand design.
}

Methods: An online survey was conducted on upper limb amputees, with 27 respondents that completed the survey. The survey was a mixture of open-ended questions, ranking design features and quantitative questions on problems experienced and desired attributes of future prosthesis designs.

Results: Common key issues and concerns were isolated in the survey related to the weight, manipulation and dexterity, aesthetics, sensory feedback and financial cost; each of which could be addressed by additive manufacturing and soft robotics techniques.

Conclusions: The adaptability of additive manufacturing and soft robotics to the highlighted concerns of participants shows that further research into these techniques is a feasible method to improve patient satisfaction and acceptance in prosthetic hands.Implications for rehabilitation Even with recent developments and advances in prosthetic design, the needs and desires of prosthetic users are not being met with current products. The desires and needs of those with upper limb difference are diverse. Using additive manufacturing to produce prosthetics allows for mass customization of prosthetics to meet these diverse needs while reducing costs. A soft robotic approach to prosthetics can help meet the desires of reducing weight and costs, while maintaining functionality.

\author{
Disciplines \\ Engineering | Science and Technology Studies
}

\section{Publication Details}

Stephens-Fripp, B., Walker, M., Goddard, E. \& Alici, G. (2019). A survey on what Australians with upper limb difference want in a prosthesis: justification for using soft robotics and additive manufacturing for customized prosthetic hands. Disability and Rehabilitation: Assistive Technology, Online First 1-8. 


\section{A Survey on What Australian's with Upper Limb Difference Want in a Prosthesis: Justification for using Soft Robotics and Additive Manufacturing for Customised Prosthetic Hands}

Benjamin Stephens-Fripp ${ }^{1,5}$, Mary Jean Walker ${ }^{2,5}$, Eliza Goddard ${ }^{3,4,5}$, and Gursel Alici, ${ }^{1,5}$.

${ }^{1}$ B. Stephens-Fripp, and G. Alici (corresponding author) are with the School of Mechanical, Materials, Mechatronic and Biomedical Engineering, University of Wollongong, Wollongong NSW 2522 Australia (gursel@uow.edu.au)

${ }^{2}$ M. Walker is with the School of Philosophical, Historical and International Studies, Monash University, Clayton VIC 3800, Australia

${ }^{3}$ E. Goddard is with the School of Humanities and Languages, University of New South Wales, Sydney NSW 2052; and ${ }^{4}$ School of Humanities, University of Tasmania, Hobart TAS 7005, Australia

${ }^{5}$ All authors are part of the ARC Centre of Excellence for Electromaterials Science (ACES)

This is an Accepted Manuscript of an article published by Taylor \& Francis Group in Disability and Rehabilitation in February 2019, available online: https://doi.org/10.1080/17483107.2019.1580777 


\title{
A Survey on What Australians with Upper Limb Difference Want in a Prosthesis: Justification for using Soft Robotics and Additive Manufacturing for Customised Prosthetic Hands
}

\begin{abstract}
Upper limb prostheses are part of a rapidly changing market place. Despite development in device design, surveys report low levels of uptake and dissatisfaction with current prosthetic design. In this study, we present the results of a survey conducted with people with upper limb difference in Australia on their use of current prostheses and preferences in a prosthetic in order to inform future prosthetic hand design. We isolate common key issues/concerns raised in the survey relevant to the design of a prosthetic hand - weight, manipulation and dexterity, aesthetics, sensory feedback, and financial cost - and show how each could be addressed by additive manufacturing and soft robotics techniques. The adaptability of these techniques to these concerns shows that further research and development into additive manufacturing and soft robotics is a feasible method to improve patient satisfaction and acceptance in prosthetic hands.
\end{abstract}

Keywords: Prosthetics; Additive Manufacturing; Soft Robotics

\section{Introduction}

There is a range of estimates for prosthetic rejection rates, with estimates of up to as high as $40 \%$ [1]. Further, $82 \%$ of upper limb prosthetic device owners use their devices in grasping-related tasks half of the time or less [2]. There have been several surveys conducted on upper limb prostheses regarding their rejection $[1,3,4,5,6]$, views on control mechanisms [7] and sensory feedback [8, 9], as well as the embodiment of prosthetic devices for amputees [10]. However, thorough surveys conducted on prosthetic user populations $[2,5,11,12]$ are quite old relative to developments in materials and engineering techniques. In the last six years, surveys have focused on specific questions (e.g. on novel control techniques [7]) and the most recent survey [13], whilst providing 
free response feedback from seven upper limb amputees, focussed on their challenges and difficulties faced throughout the day. Without any structured questions, it relied on common themes being raised by the respondents and was therefore unable to provide any quantitative information, such as ranked lists of difficulties and design priorities etc. When examining the views of those with limb difference, similarly other previous surveys have focussed on the reasons for dissatisfaction with current prosthetics and rejection, with minimal attention paid to the preferences of users of prosthetic devices. Instead of inferring what users want from questions about dissatisfaction, this present survey incorporated questions about preferences from those with upper limb difference ,including open ended responses, as input to shape future prosthetic designs. In addition the survey solicited responses to determine what areas soft robotics and additive manufacturing can contribute towards, \achieving the highest benefit for the users.

Upper limb prosthetic devices are part of a rapidly changing market with many recent developments $[14,15]$, particularly in multiple digit prostheses and advances in their design, manufacturing, control and performance. Many of these developments are not yet close to market suitability to meet needs and concerns of people with upper limb difference associated with current upper limb prosthetic devices [14, 15]. Additive manufacturing is an enabling technology to create cost effective [16], lightweight [17], and customisable [18] prosthetics.

Soft robotics is an emerging research area $[19,20,21,22,23]$ which provides an advantage of being adaptable and conformable to unknown environments through the use of lightweight and soft components with programmable compliance. Through strategic design of the intrinsic properties of the chosen soft materials, soft robotics can be used to mimic parts of some biological organisms by incorporating a one-piece compliant mechanism with flexible bending points to undergo large deformations and adapt to the 
shape of the object being gripped or interacted with, rather than using multiple rigid components and joints as used in traditional robotics [20]. Although soft robotic devices have been manufactured by moulding methods [24, 25], with strategic design and material selection, soft robotic devices can be manufactured using additive manufacturing processes [26, 27]. This reduces manufacturing costs and allows the creation of complex geometries by shortening the path from ideation to the proof of concept [20]. Both soft robotics and additive manufacturing offer great potential for the field of prosthetic devices and could meet some of the needs and desires of those with upper limb difference. Given rates of uptake and expressed dissatisfaction with current prosthetics, it is reasonable to assume that some of the issues are related to prosthetic design could be addressed by emerging techniques such as soft robotics and additive manufacturing.

There are three aims that this study seeks to address:

1) Provide a more recent survey focused on people with upper limb difference within Australia where researchers are located and where the last survey conducted was in 2002 [2].

2) Receive direct input from potential users such that this input can help shape the future direction of design and fabrication of upper limb prostheses.

3) Assess which of these current problems, desires and needs identified from the participants survey responses can be addressed using soft robotics and additive manufacturing technologies in the development of upper limb prostheses.

\section{Materials and Methods}

An online survey was created to obtain feedback from people with upper limb difference about their experiences of and desires for prosthetics using the Qualtrics 
Insight platform. An interdisciplinary research team at the ARC Centre of Excellence in Electromaterials Science (ACES) with the expertise in bioethics, engineering and biomedical device design, particularly advanced prosthetic design undertook this research. Questions were related to functionality, appearance and sensory feedback. Questions on the impact of a prosthesis on work and daily life, relationships and identity were also asked but those results and analysis will be reported on elsewhere and are not discussed within this manuscript. In addition, information about age, sex, reason for limb difference and time since limb loss was requested. Open ended free response text questions were used when feasible, and questions were phrased as neutrally as possible to reduce any unintended guidance to the response. Participants, who were people with upper limb difference, were asked to respond to quantitative questions such as ranking the features they would like to see in the future prosthetic hands, and to provide qualitative feedback on problems experienced and desired attributes of prosthetics. The results are displayed in a combination of graphs and tables, followed by a discussion of the common key points raised in the responses, and their implications for various aspects of prosthesis design. One aim of this manuscript is to assess which of these desired improvements and changes to prosthetic devices can be addressed by using the soft robotic and/or additive manufacturing approach. Refinements and alterations were made after the survey was reviewed by people with upper limb difference and advocacy groups, including Limbs 4 Life Australia and Amputees New South Wales, as well as individual stakeholders. The survey flyer, which contained a brief description and a link to the website, was disseminated through a variety of methods; including private networks, social media, post, email and e-newsletters; and distributed via a range of networks including Australian amputee and limb difference support and advocacy groups, rehabilitation clinics and hospitals, authors' universities, and professional 
associations of prosthetists, orthotists, occupational therapy and rehabilitation workers.

The survey was open to participants for 6 months during 2017. IP addresses were

collected by our software to prevent repeat submissions.

Table 1 - Survey Participant Breakdown (AE: Above Elbow, BE: Below Elbow)

\begin{tabular}{|c|c|c|}
\hline \multicolumn{3}{|c|}{ Participants (n (\%)) } \\
\hline Sex & Female & $17(62.9)$ \\
\hline & Male & $9(33.3)$ \\
\hline & Not disclosed & $1(3.7)$ \\
\hline Age in years (average) & 40.8 & \\
\hline \multirow[t]{8}{*}{ Limb difference } & Unilateral right $\mathrm{AE}$ & $6(22.2)$ \\
\hline & Unilateral left $\mathrm{AE}$ & $2(7.4)$ \\
\hline & Unilateral right $\mathrm{BE}$ & $5(18.5)$ \\
\hline & Unilateral left BE & $5(18.5)$ \\
\hline & Bilateral (left AE, right AE) & $1(3.7)$ \\
\hline & Bilateral (left BE, right BE) & $5(18.5)$ \\
\hline & Partial hands and fingers & $1(3.7)$ \\
\hline & Not disclosed & $2(7.4)$ \\
\hline \multirow{5}{*}{$\begin{array}{l}\text { Reasons for limb } \\
\text { difference }\end{array}$} & Congenital & $7(26.9$ \\
\hline & Injury or trauma & $5(19.2)$ \\
\hline & Cancer & $5(19.2)$ \\
\hline & \begin{tabular}{|l|} 
Sepsis/infection \\
\end{tabular} & $8(30.8)$ \\
\hline & Complications from surgery & $1(3.8)$ \\
\hline \multirow[t]{8}{*}{ Time since limb loss ${ }^{1}$} & Less than 3 months & 0 \\
\hline & 3-6 months & $1(6.7)$ \\
\hline & 6 months -1 year & $1(6.7)$ \\
\hline & $1-2$ years & $3(20)$ \\
\hline & $3-5$ years & $4(26.7)$ \\
\hline & $5-10$ years & $2(13.3)$ \\
\hline & $10-20$ years & $4(26.7)$ \\
\hline & More than 20 years & 0 \\
\hline
\end{tabular}


Table 2 - Breakdown of types of prosthetic devices used by participants

\begin{tabular}{|l|c|}
\hline \multicolumn{2}{|c|}{ Types of prosthetics used by survey participants (n (\%)) } \\
\hline None & $12(44.4)$ \\
\hline Cosmetic & $2(7.4)$ \\
\hline Hook & $5(18.5)$ \\
\hline Body powered & $5(18.5)$ \\
\hline Myoelectric & $7(18.5)$ \\
\hline Bebonic & $1(3.7)$ \\
\hline COAPT system & $1(3.7)$ \\
\hline Straps to hold implements & $1(3.7)$ \\
\hline Not disclosed & $1(3.7)$ \\
\hline
\end{tabular}

N.B. Total > $100 \%$ due to multiple prosthetics or prosthetics that fit into more than one category

Informed consent was obtained from each individual when they began the survey, and ethical approval was obtained from Monash University Human Research Ethics Committee. Qualitative analysis of any free response answers was undertaken independently by two of the researchers (MW and BSF), followed by a discussion for consensus on interpretation. Any groupings of key points are tabulated in decreasing order of the number of participants that it was raised by.

\subsection{Limitations}

35 responses were received, but those who only provided demographic data were removed. In total, there were 27 respondents that completed the survey and the participant breakdown is shown in table 1. Participants' responses were still retained if they skipped the free-text section or occasional questions. This small convenience sample is a relatively small proportion of the population and is unlikely a representative sample (due to the dissemination method, and as indicated by the low average age and high proportion of female participants). However, the authors propose that these results are still able to provide useful feedback on the current experiences and desires of people with upper limb difference. In order to maintain our focus on the Australian context, the authors chose not 
to open it up to those residing in another country to increase the sample size.

Even though we consulted with people with limb difference in designing the survey, this consultation could have been more extensive. Ideally, we could have included people with limb difference as co-designers and analysers. Further, while we aimed to phrase questions neutrally and in ways that did not constrict or frame potential responses, in view of responses received this may not always have succeeded. For instance, in the question on preferences about look, we included in parentheses "e.g., lifelike, uncovered or 'skeletal', customised design, other" with the intention of making the question clear. However, this may have influenced people to choose one of these responses.

As the survey was guided by the specific aims noted above, and to avoid unintentionally guiding responses, it is not intended to provide comprehensive information on preferences about all aspects of prosthetic design or manufacturing. For example, no specific questions were asked regarding the socket and/or its interaction with the residual limb, although it would presumably help contribute to a participant's perception of comfort. In addition, no questions were asked regarding the incorporation of a wrist into a design, but it did come up through other free text responses regarding functionality and dexterity.

\section{Results}

Figures 1-2 and tables 3-5 provide a collation of the results of the questions about participants' current experiences of their prosthetic device(s). Figure 1 shows average daily usage of their device. 


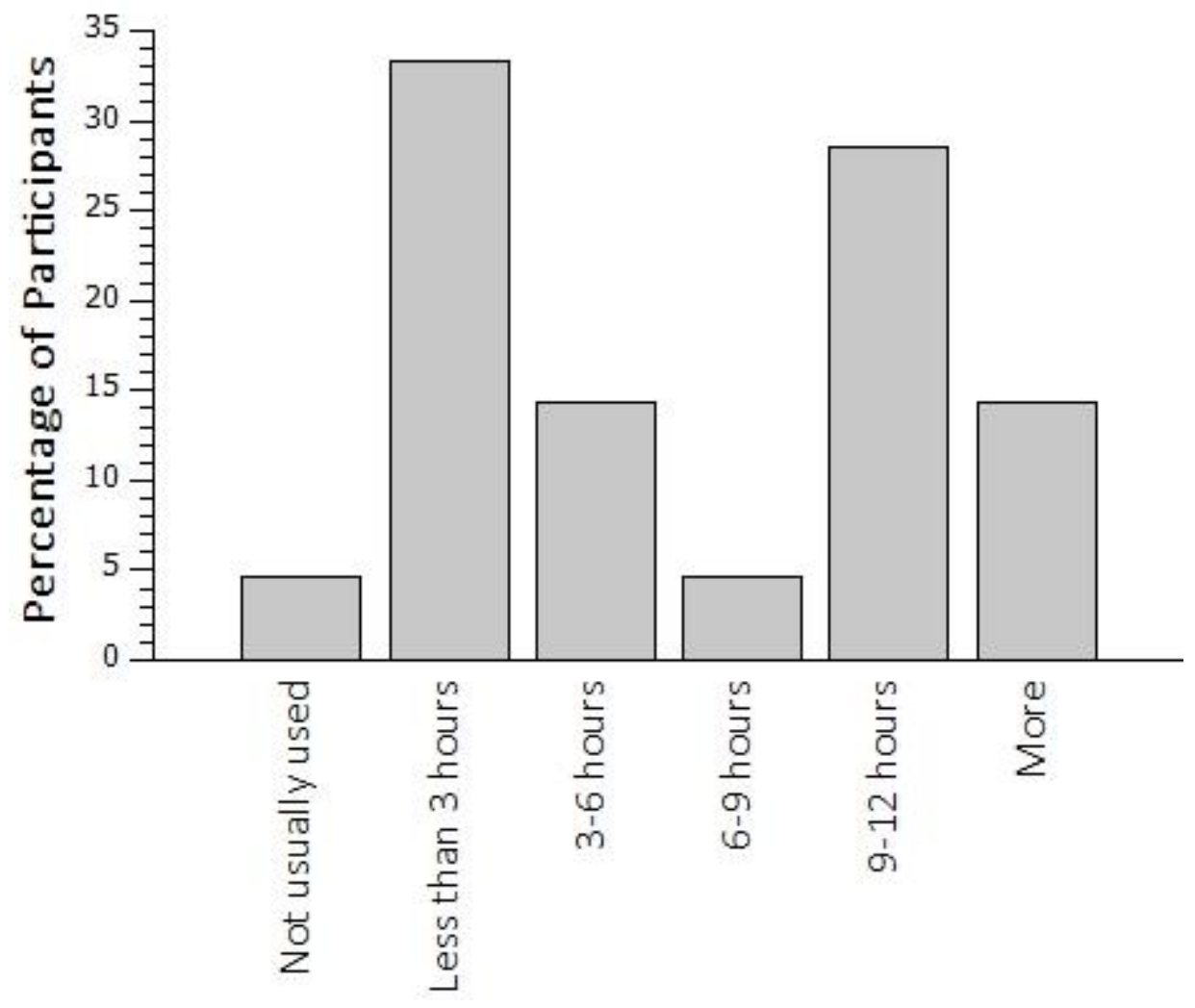

Figure 1 - Average Hours of Prosthetic Use

In a follow up question, $84.7 \%$ of respondents indicated that there are specific times or activities that they prefer not to wear a prosthetic device. Their reason was provided as an open text response and the authors identified three common key points, as shown in table 3. These were placed in descending order based on the number of times they were mentioned by the participants, and participants responses sometimes contributed to more than one of them. Participants raised some issues associated with (i) size and weight: the prosthetic was "heavy and clunky" or "too long and too heavy and awkward", and (ii) functionality and dexterity: the prosthetic lacked "wrist flexion" or caused "clumsiness". 
Table 3 - Reasons for not Wearing Prosthetic, in descending order.

\begin{tabular}{|c|l|c|}
\hline & \multicolumn{1}{|c|}{ Key Point } & Number of Participants \\
\hline 1$)$ & Size and Weight & 4 \\
\hline 2$)$ & Functionality and Dexterity & 2 \\
\hline 3$)$ & No need to use the prosthetic & 4 \\
\hline
\end{tabular}

In addition, $66.7 \%$ of the participants indicated that they had changed the kind of prosthesis they use. Their reasons centred around, for example, the prosthetic was "heavy and clunky" or "too long and too heavy and awkward", the prosthetic lacked "wrist flexion" or caused "clumsiness". These reasons were collated by the authors and ranked in descending order based on how common these key points appeared within the responses, some answers contained multiple key points.

Table 4 - Reasons for Changing Prosthetic, in descending order.

\begin{tabular}{|c|l|c|}
\hline & \multicolumn{1}{|c|}{ Key Point } & Number of Participants \\
\hline 1$)$ & Functionality and dexterity & 1 \\
\hline 2$)$ & Size and weight & 1 \\
\hline 3$)$ & Reliability & 1 \\
\hline 4$)$ & Financial capacity & 1 \\
\hline 5$)$ & Altered self-understanding & 1 \\
\hline 6$)$ & Look & \multicolumn{2}{|c|}{} \\
\hline
\end{tabular}

Participants were asked to identify issues with their current prosthesis in a free text response. These responses were grouped together in common key points and are presented in Table 5. Each participant's response can contribute to more than one key point. 
Table 5 - Problems with Current Prosthesis, in descending order.

\begin{tabular}{|l|l|c|}
\hline & \multicolumn{1}{|c|}{ Key Point } & Number of Participants \\
\hline 1$)$ & Limited functionality & 4 \\
\hline 2$)$ & Size and weight & 3 \\
\hline 3$)$ & Reliability & 2 \\
\hline 4$)$ & Ease of use and comfort & 1 \\
\hline 5$)$ & Design trade-offs/problems & 3 \\
\hline
\end{tabular}

Similar to responses in previous surveys $[4,13]$, limited functionality and the large size and weight of current prosthetics are still listed as the largest problem with current prosthesis.

Figure 2 breaks down the source of funding used for purchasing each participant's prosthetic device.

\section{Funding Source}

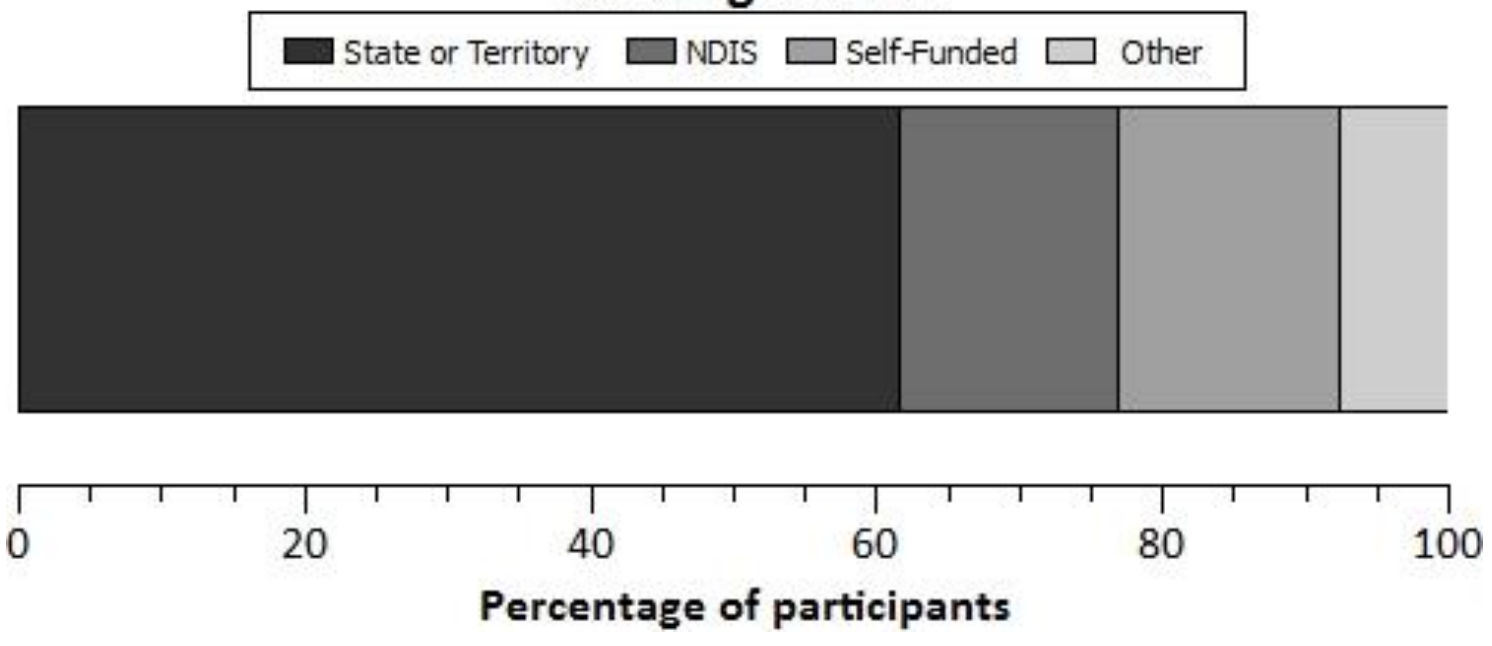

Figure 2 - Payment method for participants' most recent prosthetic. N.B. National Disability Insurance Scheme (NDIS) is a scheme in which the Australian government provides support for those with a permanent and significant disability to its citizens and permanent residents. 
Tables 6 and 7 outline the type of activities and grasping tasks that participants reported were most important to be able to use their prosthetic device for.

Participants were asked what activities were most important to them to be able to do them with a prosthetic device. Their responses were given in a free text response and were collated and ranked in descending order based on how commonly these key points appeared within the free text responses, some responses contained multiple key points within them. These key points are presented in Table 6.

Table 6 - Most Important Activities for Prosthesis, in descending order.

\begin{tabular}{|l|l|c|}
\hline & \multicolumn{1}{|c|}{ Key Point } & Number of Participants \\
\hline 1$)$ & Activities of daily living & 13 \\
\hline 2$)$ & Sport and leisure activities & 6 \\
\hline 3$)$ & Writing/Typing & 6 \\
\hline 4$)$ & Driving & 5 \\
\hline
\end{tabular}

When participants were asked if there were tasks they currently did not use their prosthetic device for, many of the respondents indicated tasks such as everything presented in the previous question (shown in Table 6), or "so much more", indicating a large non-satisfaction with current performance of prosthetics. In addition to using terms such as those shown in table 6, responses included statements such as enabling tasks such as eating, holding mobile phone, handling a wallet, cooking, being able to hold their children.

Table 7 presents a collated list of most desirable grasping types by the participants. They were asked to provide the most important grasping and manipulation tasks for them in a free response form. The authors classified responses as indicating a desire for reliable 
grasp where a participant simply indicated wanting to be able to have and keep hold of an object (e.g., "holding [...] cans, jars" or "steadying objects"); a strong grip where strength was specifically mentioned or indicated as desired (e.g., "strong secure grip" and for "picking up heavy items"); and a precise grip where the response indicated a desire to control grip strength (e.g., "touch grip strength so doesn’t crush things").

Table 7 - Most important grasping and manipulation tasks, in descending order

\begin{tabular}{|c|l|c|}
\hline & \multicolumn{1}{|c|}{ Key Point } & Number of Participants \\
\hline 1$)$ & Fine and Delicate tasks & 5 \\
\hline 2$)$ & Reliable Grasp/Hold & 5 \\
\hline 3$)$ & Strong Grip & 3 \\
\hline 4$)$ & Precisely Controlled Grip & \\
\hline
\end{tabular}

Participants were asked to rank the importance of various design features using a sliding bar scale of $0-5$, with 0 being the least important and 5 being the most important. If a participant did not provide a response for one of the features, it was not considered in the calculations. Figure 3 shows the average rating for each of these factors.

In addition, participants were asked what factors determine their choice in prosthesis selection. The top three choices are shown in figure 4.

Three participants also selected an "other" option, indicating preference of comfort, strength and weight being high priorities. 


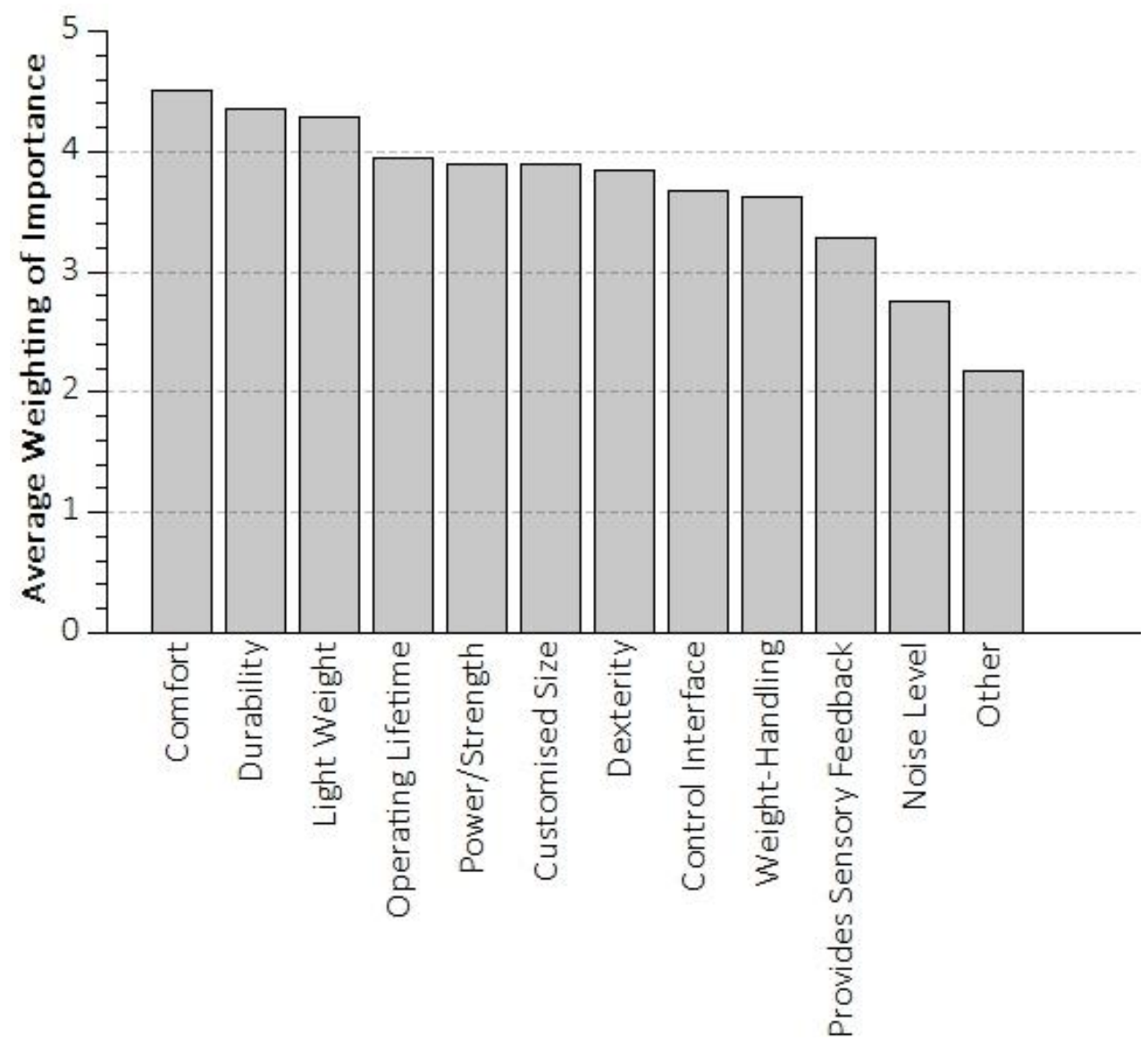

Figure 3 - Average Weightings of importance

Participants were asked to identify their preference for how they would like their prosthesis to appear and provide their reason in a free response form. However, they were given the examples of "lifelike, uncovered or 'skeletal', customised design, other". They were able to provide more than one option and these were grouped together, as presented in Table 8. The only "other" option was for a robotics/cyborg look indicated by four participants. 


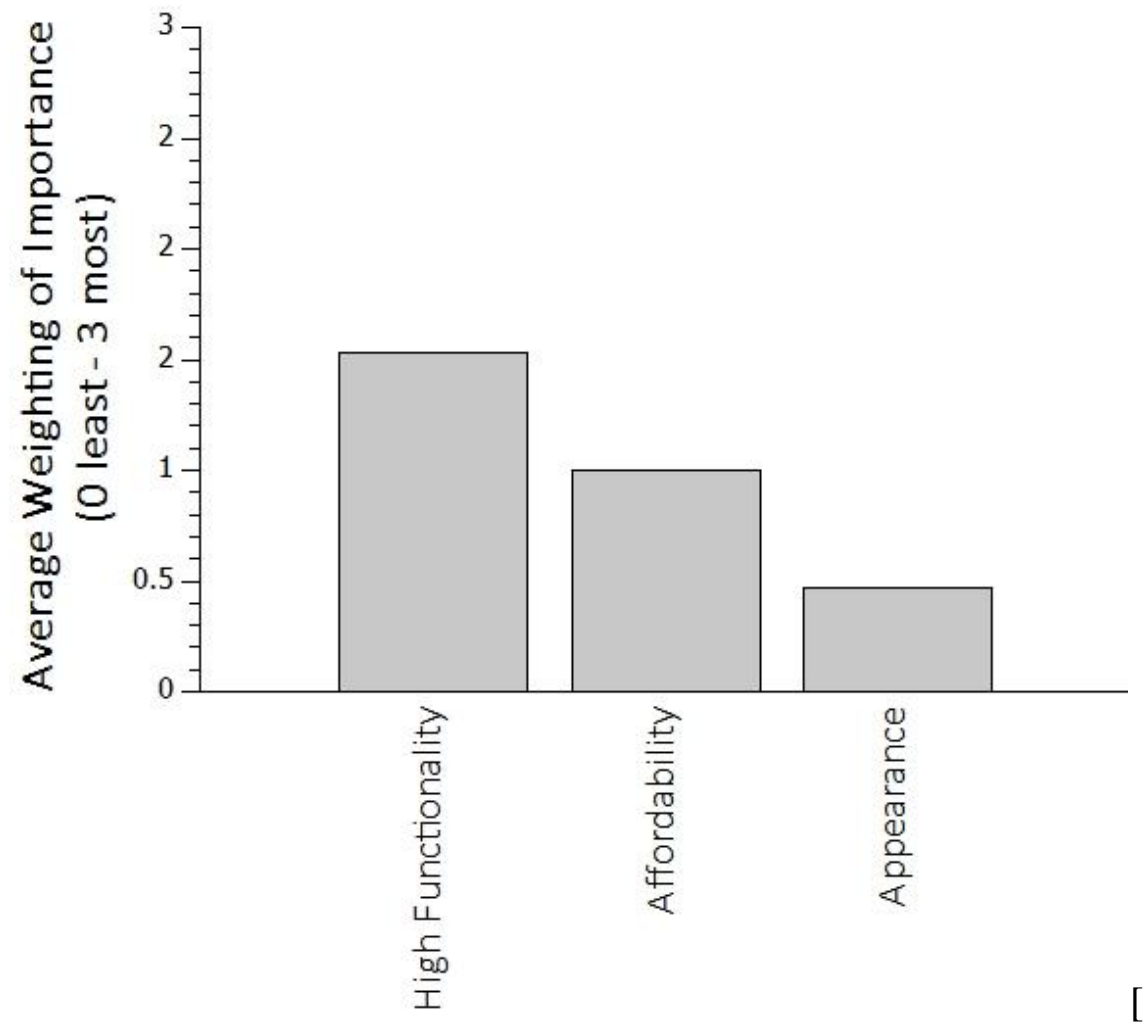

Figure 4 - Average of participant's ranking of importance of considerations in choosing a prosthetic (1 least important, to 3 - most important

Table 8 - Preference on prosthetic appearance. (N.B. some indicated that they like more than one kind of look, $\mathrm{N}=18$ )

\begin{tabular}{|l|c|}
\hline \multicolumn{1}{|c|}{ Preference } & Frequency \\
\hline Life Like & 8 \\
\hline Customisable & 3 \\
\hline Robotic/cyborg & 4 \\
\hline Skeletal/uncovered & 3 \\
\hline Appearance unimportant & 2 \\
\hline
\end{tabular}

As shown in Table 8, within this sample there was a varied level of response to aesthetic appearance. In addition, the language used was often quite strong when they described their preference of appearance (lifelike vs robotic/skeletal). E.g. one survey participant wanted their prostheses to look "lifelike so I blend in with the crowd" whereas another 
participant responded that their preference was "Definitely not lifelike" but rather a “customised design".

Participants were given a free response question to detail what sensory feedback sensations would be desirable for them. The results are grouped into common key points in Table 9. Some participants responded with more than one type of sensation, whereas responses of multiple participants were either left blank or indicated they were "not sure". This may indicate that some participants have not considered how it would be helpful, particularly if they have fundamental problems with their prosthetic that caused them to give up using it all together, and it may be difficult for them to imagine both it is being useful and what it would be like [28].

Table 9 - Participants' interest in different types of sensory feedback

\begin{tabular}{|l|c|}
\hline \multicolumn{1}{|c|}{ Sensory Feedback } & Frequency \\
\hline Grip Strength & 7 \\
\hline Feeling of touch & 5 \\
\hline Temperature & 1 \\
\hline Texture & 1 \\
\hline Not sure & 2 \\
\hline Not interested in sensory feedback & 1 \\
\hline
\end{tabular}

One avenue for application of natural and efficient non-invasive sensory feedback is through the use of Phantom Limb Maps [29, 30, 31, 32, 33] from those who have undergone amputation. Phantom digits or a phantom hand map is a phenomenon where touching a specific area on the residual limb causes the feeling that one of the missing fingers is being touched. However, these phantom digits are not experienced by all amputees and their locations can vary. Their prevalence is also unknown amongst those with upper limb difference. Within the participants who had undergone amputation, i.e. 
excluding those with congenital limb difference, $47 \%$ indicated that they had at least one of these phantom digits. This is lower than the previously reported value of 12 out of 18 participants (66\%) reported by Ehrsson et al. [34]. It may be possible that the other participants are unaware of this phenomenon and/or have not discovered it yet or may have more spots not identified if they are smaller regions. It is worth noting that both of these studies have very small sample sizes so are not a reliable indication of the wider population. For the participants who indicated they had phantom digits, Figure 5 provides a breakdown of the number of corresponding digits for which this phenomenon occurred, as well as a breakdown of the number of times each digit had a corresponding phantom digit for nine participants.

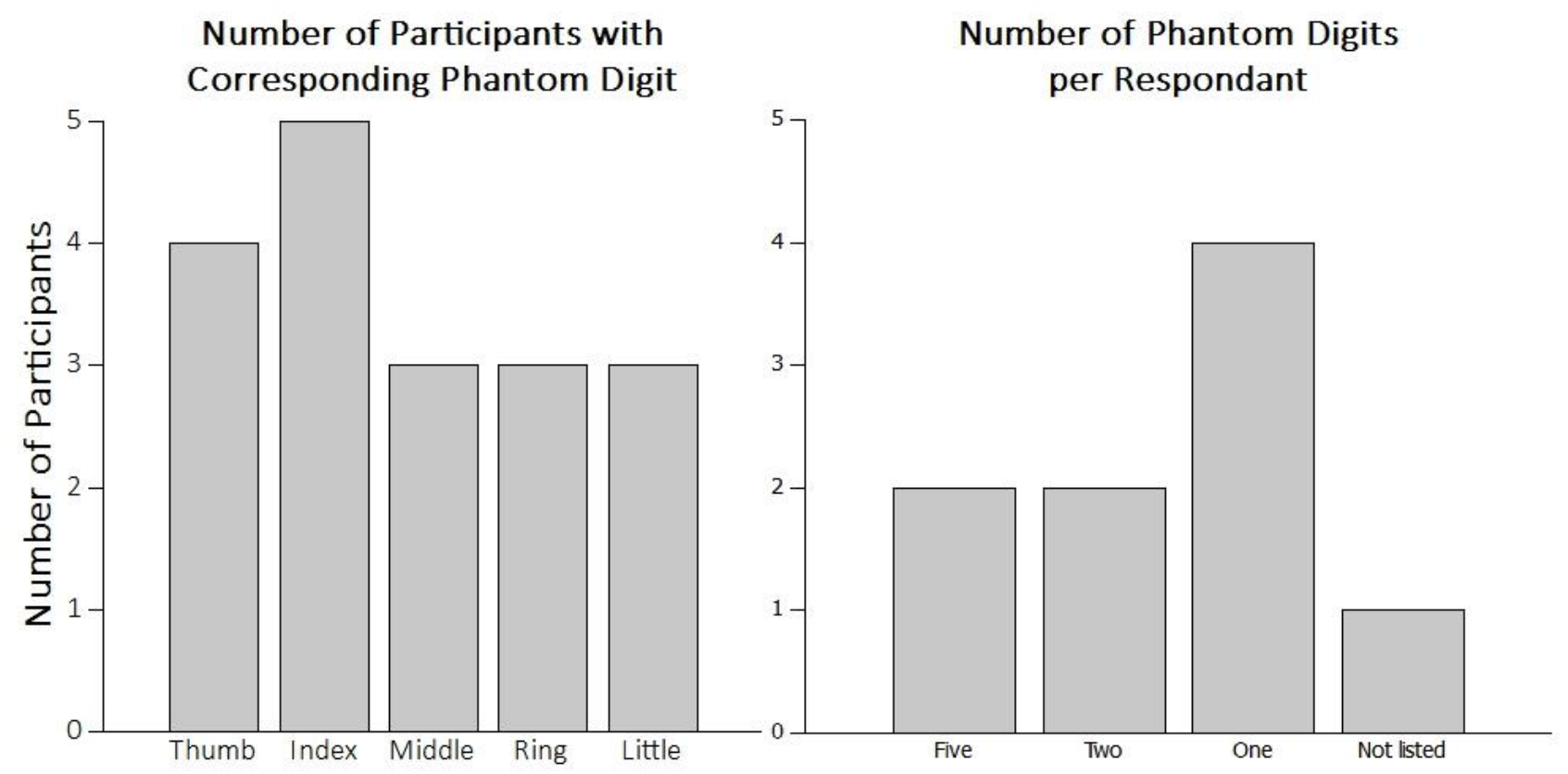

Figure 5 - Phantom digits breakdown from those who indicated them

The largest benefit to using phantom digits as a site for sensory feedback comes from being able to easily distinguish between multiple sites and correctly associate them without training or learning of substitution feedback methods. However, four out of nine 
participants indicated they only had one phantom digit, which minimises any advantage it may have over substitute sensory feedback.

\section{Discussion}

The average time that participants use their prosthetic device each day is shown in Figure 1. Consistent with previous surveys $[1,2]$, our data shows that there is still an under usage of prosthetic devices, with $52.3 \%$ of individuals who owned a prosthetic using their device for less than 6 hours per day. This indicates that users, are still either not satisfied or do not take themselves to derive sufficient benefit from their current prosthetic device. Given our survey responses, it is reasonable to assume that considerations in design may be a factor in this dissatisfaction. Therefore, the key design points of weight, manipulation and dexterity, sensory feedback, aesthetics and costs will be analysed in this section. A recurrent idea throughout these sections is that both a soft robotic approach (using soft materials to establish conformable, light weight, low cost and low foot-print devices, here typified by prosthetic hands) to prosthetic devices and additive manufacturing (for ease of customisation and reduction in cost) can be expected to improve prosthetic devices in many of the ways potential users desire.

\subsection{Weight}

As shown in Figure 3, weight is very important in design features of prosthetics. By itself it ranks third, and it also contributes to the comfort of a prosthesis, ranked first. That the current weight of prosthetic hands is a major issue needing improvement in the establishment of new prosthetic devices was further borne out by participants' free text responses as shown in Tables 3-5. Participants' responses indicated that when considering 
comfort, they experienced issues surrounding the socket connection, as well as the contribution that the weight makes to the overall comfort. The recent approach of soft robotics for prosthetic devices $[19,20,35]$ provides a possible pathway to greatly decrease the weight, whilst still enabling fine motor control and manipulation. Due to the materials' compliance and adaptability, they rely on under actuation and can conform to the object's shape, thus allowing a reduction in the number of actuators or motors required, which impacts the overall weight of the hand.

A major contributor to the weight of the hand is the battery and although the battery size may be reduced as a result of fewer actuators, it will still be a major contributor. Recently there has been success into the development of 3-D printable batteries [36], which could assist to locate the battery into the position with the minimal impact on the weight of hand, allowing it to conform to the available space and spread the weight distribution.

\subsection{Manipulation and Dexterity}

In line with the wide variety of prosthetics available in the market $[14,15]$, prosthetic hands are increasing the number of controllable digits and their dexterity. However, as shown in Figure 4, increasing functionality is still one of the most important features of the prosthetic devices. There are several factors including accessibility due to costing, weight, sensory feedback and control algorithms which should be considered together with the functionality. Although prosthetic hands currently have the ability for fine motor control, they are limited in the controllability from the user, typically through myoelectric signals. It is currently difficult to achieve proportional fine motor control through myoelectric signals. In addition, visual feedback does not provide enough information for 
appropriate control of hands [37]. Therefore, providing sensory feedback is an important part of increasing the user's ability to enable fine control, which current top-end prosthetic devices do not have. Typically, as the functionality of the hand goes up, so does the requirement for high performance actuators and other components, which affect both the weight and cost of prosthetic hands. This could be one explanation for why only $22.2 \%$ of participants in this survey owned a myoelectric prosthetic device. As discussed, employing recent progress in soft robotic approaches, using manufacturing technologies including additive manufacturing, may provide one pathway to maintain the fine dexterity required, whilst minimising cost and weight [38].

\subsection{Aesthetics}

As shown in Table 8, there is no overwhelming consensus on what kind of appearance is preferred and free-text explanations of preferences indicated that participants had strong personal reasons for their preferences. For example, some preferred a lifelike appearance as they did not want to stand out, whilst others wanted to stand out from the crowd and for people to notice their prosthetic device. Traditional manufacturing methods, however, make it difficult and expensive to provide various options. The increasing ability in developing prosthetic hands through additive manufacturing provides a pathway that will easily enable a high level of customisation for users, not only in their look but also in the appropriate sizing, whilst minimising cost and weight. Taking an additive manufacturing approach also enables possibilities for distributed/on-site manufacturing for prosthetic fitters and to provide easier access, particularly in developing countries. 


\subsection{Sensory Feedback}

Although sensory feedback is listed lower on the priority list than other features, as shown in Figure 3, it still has an average weighting of 3.3 / 5. However, sensory feedback can play a significant role in achieving the desired grasps outlined in Table 7 (apart from strong grip). In addition, due to this feature not being readily available there is a lack of familiarity/knowledge of its potential capacity with some participants responding they were "not really sure" or "literally have no clue" with what sensations would be most important to them. Although there has been extensive research on sensory feedback techniques $[39,40,41,42,43]$, they have not made it to marketplace yet. There is still therefore a great need for a simple, yet practical and effective sensory feedback method.

\subsection{Costs}

Improvements in prosthetic devices can also increase cost. Affordability is a concern for current prosthetic users, as shown in Figures 2 and 4, and Table 4. This is of particular interest in the Australian context, where $76.9 \%$ of the survey's participants indicated that their funding for prosthetic device was through either a state-based or national public provision scheme, which only provides a minimal rebate to cover the cost of a basic prosthetic hand. However, this is likely to be a key consideration in many other locations too. Traditional manufacturing methods with multiple actuators (i.e., motors) per digit make a highly functional prosthetic device unattainable to most recipients due to financial constraints. However, the combination of lower production costs due to additive manufacturing and lower actuator requirements resulting from an under actuated soft robotic approach, make the goal of an affordable highly functioning prosthetic more achievable. 


\subsection{Potential Future Implications}

Due to the potential lower costs involved, a soft robotic and additive manufacturing could lead to a bigger variation in hands available for purchase and also provide the patient with the potential to afford multiple prosthetic devices. Our survey results indicate that the needs and desires of devices not only differ between users, but user needs can also change as life circumstances change. Potentially users could afford multiple devices to suit different needs in life, and easily change devices when life needs and/or circumstances change.

This approach could also lead to an expanded role of the occupational therapist and prosthetist in the building and adapting a hand to fit the user. Currently an occupational therapist helps a user determine the most suitable prosthetic device for the user and their needs, chosen from a small fixed number of models on the market. A prosthetist will then build a custom socket to fit the patient and the device, and the occupational therapist will help train them in their use. However, if a mass customisation approach is taken, more adaptability and customising is available to the prosthetic team. In addition, since additive manufacturing allows for relatively easy changes in design without significant additional production expenses, compared to traditional manufacturing, a potential future exists where the prosthetic team could alter a variety options; such as the hands shape, weight, size, colour; to help increase the comfort and acceptance of the hand for the user. In order to maximise this approach's success, it is imperative to involve prosthetists and occupational therapists in a discussion of their desires and needs for customisable options, so that the ability to change these features is built into any standard design. 


\subsection{Other areas of consideration for design}

As shown in Figure 3, comfort is still a major priority for prosthetic users. Size and weight are the major contributors to this, as discussed in section 4.1. Although not a focus of this survey, previous results indicated that the actual socket to residual limb connection was a large source of skin irritation and discomfort [1,4] and hence is another major contributor to overall comfort in prosthetic devices which requires further research and development. Osseo-integration [44] is one technique used to minimise the discomfort caused through socket interaction, whereby the socket is mechanically implanted into the bone of the residual limb. However, this is a highly invasive procedure and is not suitable for many upper limb amputees due to the unavailability of suitable bone anchorage.

Figure 4 demonstrates that durability is also a significant concern for users and it is directly associated with additive manufacturing and soft robotic approaches. Having the prosthetics completed in one print without post assembly [45] may contribute towards higher durability. In addition, if the cost is reduced, and manufacturing is able to be distributed (due to less complicated assembly) resulting in smaller repair times, this may also reduce the importance of this priority for the users. However, it is important to keep this priority in mind when designing current prostheses.

Although within each question it was of minimal significance, when examining the results as a whole, the desire for wrist flexion was a common recurrence throughout different questions in free text response from different participants. For the participants in our survey, it was seen as a key feature in improving the dexterity and usability of upper limb prosthetics. 


\section{Conclusion}

This study has presented an insight into the experiences and desires of upper limb prosthesis users in Australia. The survey evidences a high need for an affordable, light weight dexterous prosthetic hand. These goals are difficult to achieve together using traditional manufacturing approaches. Using a soft robotic approach to prosthetic hand design resulting from additive manufacturing presents a pathway to help achieve a larger number of these goals simultaneously. In addition, it allows for the possibility of mass customisation, permitting individualisation of both size and appearance, which could increase the comfort of the prosthesis as well as lead towards a greater acceptance and utilisation of the device.

\section{References}

1. E.A. Biddiss and T.T. Chau, "Upper limb prosthesis use and abandonment: A survey of the last 25 years," Prosthetics and Orthotics International, vol. 31, no. 3, 2007, pp. 236-257; DOI doi:10.1080/03093640600994581.

2. J. Davidson, "A survey of the satisfaction of upper limb amputees with their prostheses, their lifestyles, and their abilities," Journal of Hand Therapy, vol. 15, no. 1, 2002, pp. 62-70; DOI 10.1053/hanthe.2002.v15.01562.

3. K. Østlie, et al., "Prosthesis rejection in acquired major upper-limb amputees: A population-based survey," Disability and Rehabilitation: Assistive Technology, vol. 7, no. 4, 2012, pp. 294-303; DOI 10.3109/17483107.2011.635405.

4. E. Biddiss and T. Chau, "Upper-limb prosthetics: critical factors in device abandonment," American journal of physical medicine \& rehabilitation, vol. 86, no. 12, 2007, pp. 977-987.

5. P.J. Kyberd and W. Hill, "Survey of upper limb prosthesis users in Sweden, the United Kingdom and Canada," Prosthetics and Orthotics International, vol. 35, no. 2, 2011, pp. 234241; DOI 10.1177/0309364611409099.

6. F. Cordella, et al., "Literature Review on Needs of Upper Limb Prosthesis Users," Frontiers in Neuroscience, vol. 10, no. 209, 2016; DOI 10.3389/fnins.2016.00209.

7. S.M. Engdahl, et al., "Surveying the interest of individuals with upper limb loss in novel prosthetic control techniques," Journal of NeuroEngineering and Rehabilitation, vol. 12, no. 1, 2015; DOI 10.1186/s12984-015-0044-2. 
8. U. Wijk and I. Carlsson, "Forearm amputees' views of prosthesis use and sensory feedback," J Hand Ther, vol. 28, no. 3, 2015, pp. 269-277; quiz 278; DOI 10.1016/j.jht.2015.01.013.

9. S. Lewis, et al., "User demands for sensory feedback in upper extremity prostheses," Proc. MeMeA 2012 - 2012 IEEE Symposium on Medical Measurements and Applications, Proceedings, 2012, pp. 188-191.

10. T. Asai, et al., "Development of Embodied Sense of Self Scale (ESSS): Exploring Everyday Experiences Induced by Anomalous Self-Representation," Frontiers in Psychology, vol. 7, no. 1005, 2016; DOI 10.3389/fpsyg.2016.01005.

11. L.V. McFarland, et al., "Unilateral upper-limb loss: Satisfaction and prosthetic-device use in veterans and servicemembers from Vietnam and OIF/OEF conflicts," Journal of Rehabilitation Research and Development, vol. 47, no. 4, 2010, pp. 299-316; DOI 10.1682/JRRD.2009.03.0027.

12. C.H. Jang, et al., "A Survey on Activities of Daily Living and Occupations of Upper Extremity Amputees," Ann Rehabil Med, vol. 35, no. 6, 2011, pp. 907-921.

13. H.L. Benz, "Upper extremity prosthesis user perspectives on unmet needs and innovative technology," 2016, pp. 287-290.

14. M. Atzori and H. Müller, "Control Capabilities of Myoelectric Robotic Prostheses by Hand Amputees: A Scientific Research and Market Overview," Frontiers in Systems Neuroscience, vol. 9, no. 162, 2015; DOI 10.3389/fnsys.2015.00162.

15. S.G. Meek, "Prosthetic Limbs," Neuroprosthetics, 2nd ed., World Scientific, 2017, pp. 793-810.

16. K. Silva, et al., "Three-Dimensional (3-D) Printing: A Cost-Effective Solution for Improving Global Accessibility to Prostheses," PM\&R, vol. 7, no. 12, 2015, pp. 1312-1314; DOI https://doi.org/10.1016/j.pmrj.2015.06.438.

17. M.B. Burn, A. Ta and G.R. Gogola, "Three-Dimensional Printing of Prosthetic Hands for Children," The Journal of Hand Surgery, vol. 41, no. 5, 2016, pp. e103-e109; DOI https://doi.org/10.1016/j.jhsa.2016.02.008.

18. A. Mohammadi, et al., X-Limb: A Soft Prosthetic Hand with User-Friendly Interface: Proceedings of the 4th International Conference on NeuroRehabilitation (ICNR2018), October 16-20, 2018, Pisa, Italy, 2019, p. 82-86.

19. G. ALICI, "Softer is Harder: What Differentiates Soft Robotics from Hard Robotics?," MRS Advances, 2018, pp. 1-12.

20. R. Mutlu, et al., "3D printed flexure hinges for soft monolithic prosthetic fingers," Soft Robotics, vol. 3, no. 3, 2016, pp. 120-133.

21. H. Banerjee, Z. Tsz Ho Tse and H. Ren, Soft robotics with compliance and adaptation for biomedical applications and forthcoming challenges, 2018.

22. Ş. Kumbay Yıldız, R. Mutlu and G. Alici, Position Control of a Soft Prosthetic Finger with Limited Feedback Information, 2018, p. 700-705.

23. M. Cianchetti, et al., Biomedical applications of soft robotics, 2018.

24. T.M. T., et al., "A Resilient, Untethered Soft Robot," Soft Robotics, vol. 1, no. 3, 2014, pp. 213-223; DOI 10.1089/soro.2014.0008.

25. B. Mosadegh, et al., "Pneumatic Networks for Soft Robotics that Actuate Rapidly," Advanced Functional Materials, vol. 24, no. 15, 2014, pp. 2163-2170; DOI doi:10.1002/adfm.201303288.

26. J. Gul, et al., 3D printing for soft robotics - a review, 2018, p. 243-262.

27. T. Wallin, J. Pikul and R. F. Shepherd, 3D printing of soft robotic systems, 2018, p. 1. 28. C.D. Murray, "An interpretative phenomenological analysis of the embodiment of artificial limbs," Disability and Rehabilitation, vol. 26, no. 16, 2004, pp. 963-973; DOI 10.1080/09638280410001696764. 
29. A. Bjorkman, et al., "Sensory Qualities of the Phantom Hand Map in the Residual Forearm of Amputees," J. Rehabil. Med., vol. 48, no. 4, 2016, pp. 365-370; DOI 10.2340/16501977-2074.

30. G.H. Chai, et al., "Phantom finger perception evoked with transcutaneous electrical stimulation for sensory feedback of prosthetic hand," Proc. 2013 6th International IEEE/EMBS Conference on Neural Engineering (NER), 2013, pp. 271-274.

31. G. Chai, et al., "Characterization of evoked tactile sensation in forearm amputees with transcutaneous electrical nerve stimulation," J Neural Eng, vol. 12, no. 6, 2015, pp. 066002; DOI 10.1088/1741-2560/12/6/066002.

32. C. Antfolk, et al., "Artificial redirection of sensation from prosthetic fingers to the phantom hand map on transradial amputees: vibrotactile versus mechanotactile sensory feedback," IEEE Trans Neural Syst Rehabil Eng, vol. 21, no. 1, 2013, pp. 112-120; DOI 10.1109/TNSRE.2012.2217989.

33. C. Antfolk, et al., "Artificial Redirection of Sensation From Prosthetic Fingers to the Phantom Hand Map on Transradial Amputees: Vibrotactile Versus Mechanotactile Sensory Feedback," IEEE Transactions on Neural Systems and Rehabilitation Engineering, vol. 21, no. 1, 2013, pp. 112-120; DOI 10.1109/TNSRE.2012.2217989.

34. H.H. Ehrsson, et al., "Upper limb amputees can be induced to experience a rubber hand as their own," Brain, vol. 131, no. Pt 12, 2008, pp. 3443-3452; DOI 10.1093/brain/awn297.

35. A. Ajoudani, et al., "Exploring teleimpedance and tactile feedback for intuitive control of the Pisa/IIT SoftHand," IEEE Trans Haptics, vol. 7, no. 2, 2014, pp. 203-215; DOI 10.1109/TOH.2014.2309142.

36. C. Reyes, et al., "Three-Dimensional Printing of a Complete Lithium Ion Battery with Fused Filament Fabrication," ACS Applied Energy Materials, vol. 1, no. 10, 2018, pp. 52685279; DOI 10.1021/acsaem.8b00885.

37. R.S. Johansson and J.R. Flanagan, "Coding and use of tactile signals from the fingertips in object manipulation tasks," Nat Rev Neurosci, vol. 10, no. 5, 2009, pp. 345-359; DOI $10.1038 / \mathrm{nrn} 2621$.

38. R. Deimel and O. Brock, "A novel type of compliant and underactuated robotic hand for dexterous grasping," The International Journal of Robotics Research, vol. 35, no. 1-3, 2016, pp. 161-185; DOI 10.1177/0278364915592961.

39. B. Stephens-Fripp, G. Alici and R. Mutlu, "A Review of Non-Invasive Sensory Feedback Methods for Transradial Prosthetic Hands," IEEE Access, 2018.

40. G.W. Vidal, et al., "Review of Brain-Machine Interfaces Used in Neural Prosthetics with New Perspective on Somatosensory Feedback through Method of Signal Breakdown," Scientifica (Cairo), vol. 2016, 2016, pp. 8956432; DOI 10.1155/2016/8956432.

41. D. Perruchoud, et al., "Biomimetic rehabilitation engineering: the importance of somatosensory feedback for brain-machine interfaces," J Neural Eng, vol. 13, no. 4, 2016, pp. 041001; DOI 10.1088/1741-2560/13/4/041001.

42. R.A. Normann and E. Fernandez, "Clinical applications of penetrating neural interfaces and Utah Electrode Array technologies," J Neural Eng, vol. 13, no. 6, 2016, pp. 061003; DOI 10.1088/1741-2560/13/6/061003.

43. B.T. Nghiem, et al., "Providing a sense of touch to prosthetic hands," Plast Reconstr Surg, vol. 135, no. 6, 2015, pp. 1652-1663; DOI 10.1097/PRS.0000000000001289.

44. M. Ortiz-Catalan, B. Hakansson and R. Branemark, "An osseointegrated humanmachine gateway for long-term sensory feedback and motor control of artificial limbs," Sci Transl Med, vol. 6, no. 257, 2014, pp. 257re256; DOI 10.1126/scitranslmed.3008933.

45. J. Cuellar, et al., Ten guidelines for the design of non-assembly mechanisms: The case of 3D-printed prosthetic hands, 2018, p. 095441191879473. 